\title{
A Tightly Coupled Positioning Solution for Land Vehicles in Urban Canyons
}

\author{
$\mathrm{Xu} \mathrm{Li},{ }^{1}$ Rong Jiang, ${ }^{1}$ Xianghui Song, ${ }^{2}$ and $\mathrm{Bin} \mathrm{Li}^{2}$ \\ ${ }^{1}$ School of Instrument Science and Engineering, Southeast University, Nanjing, China \\ ${ }^{2}$ Key Laboratory of Technology on Intelligent Transportation Systems, Ministry of Transport, \\ Research Institute of Highway Ministry of Transport, Beijing 100088, China \\ Correspondence should be addressed to Xu Li; lixu.mail@163.com
}

Received 14 November 2016; Revised 11 January 2017; Accepted 6 February 2017; Published 5 March 2017

Academic Editor: Pietro Siciliano

Copyright (c) $2017 \mathrm{Xu} \mathrm{Li}$ et al. This is an open access article distributed under the Creative Commons Attribution License, which permits unrestricted use, distribution, and reproduction in any medium, provided the original work is properly cited.

\begin{abstract}
The integration between Global Navigation Satellite System (GNSS) and on-board sensors is widely used for vehicle positioning. However, as the main information source in the integration, the positioning performance of single- or multiconstellation GNSSs is severely degraded in urban canyons due to the effects of Non-Line-Of-Sight (NLOS) and multipath propagations. How to mitigate such effects is vital to achieve accurate positioning performance in urban canyons. This paper proposes a tightly coupled positioning solution for land vehicles, fusing dual-constellation GNSSs with other low-cost complementary sensors. First, the nonlinear filter model is established based on a cost-effective reduced inertial sensor system with 3D navigation solution. Then, an adaptive fuzzy unscented Kalman filter (AF-UKF) algorithm is developed to achieve the global fusion. In the implementation of AF-UKF, the fuzzy calibration logic (FCL) is designed and introduced to adaptively adjust the dependence on each received satellite measurement to effectively mitigate the NLOS and multipath interferences in urban areas. Finally, the proposed solution is evaluated through experiments. The results validate the feasibility and effectiveness of the proposed solution.
\end{abstract}

\section{Introduction}

The importance of accuracy and integrity in a positioning system has increasingly been emphasized in many intelligent transportation system or intelligent vehicle applications such as advanced driver assistance systems, intersection collision warnings, and traffic control [1-3]. During the past years, the satellite-based positioning system like the Global Positioning System (GPS) has become the primary solution for vehicle navigation. However, in critical environments such as urban canyons, the satellite signal is often blocked and/or reflected by surrounding objects causing multipath and Non-LineOf-Sight (NLOS) signal interferences $[4,5]$. The multipath means that the direct path and reflected path are received together. In contrast, the NLOS signal is only received via reflection, that is, no direct Light of Sight (LOS) path exists. In such environments, the standalone GPS cannot guarantee an accurate and continuous positioning.

To solve the issues mentioned above, there have been a number of techniques presented in the literature. Among them, the fusion of GPS with inertial navigation system (INS) and/or odometer and so forth has been a common one due to their complementary natures [6-8]. To fuse the information from multiple sensors, the Kalman filter and extended Kalman filter (EKF) are widely utilized [9-11]. Although the EKF-based methods can provide an efficient performance in many practical applications, its linearization process may suffer from divergence due to approximations during the linearization process and system mismodelling. Besides, note that nowadays, only the low-cost INS based on microelectromechanical systems (MEMS) is affordable for land vehicle applications [12, 13]. However, such low-cost INS and odometer errors diverge rapidly over time, and thus the fusion above still cannot provide acceptable navigation performance for long GPS signal outages or in the event of considerable NLOS delays.

With the rapid development of other Global Navigation Satellite Systems (GNSS) such as GLONASS and BeiDou, a possible way to fill this gap is to adopt the multiconstellation approach [14-16]. Just like GPS, GLONASS has also been 
fully operational. BeiDou attained initial regional operational status at the end of December 2011. It can now provide positioning, navigation, and timing services in the whole Asia-Pacific region and is expected to be fully operational in 2020. The combination of GPS, BeiDou, and other satellite constellations will provide far superior satellite geometry and signal availability compared to GPS alone. However, due to the serious impact of NLOS and multipath on the positioning accuracy in urban areas, such improvements in the availability of satellite signals do not necessarily facilitate high-precision positioning at the same time.

A series of technologies has been developed to mitigate the multipath and NLOS effects. For the multipath, they are mainly cataloged into three: antenna-based, receiverbased, and navigation-processor-based techniques [17-20]. Generally speaking, the multipath can be effectively mitigated via such techniques (the error is less than few meters). In contrast, the NLOS signals have more severe impact (can cause the positioning error as large as a few hundred meters) and are difficult to be discriminated in urban canons. In recent years, various $3 \mathrm{D}$ maps have been proposed and constructed to detect, exclude, or compensate NLOS delays $[4,21-24]$. Though viable and useful, it is prerequisite to measure and map the building height information of the driving environments beforehand. Moreover, it may lead to huge errors if the constructed 3D map has not enough accuracy. Generally, how to mitigate the NLOS effect to improve the positioning result is still an open research topic.

In this paper, we propose a tightly coupled positioning solution for land vehicles in urban environments, which fuses low-cost complementary sensors including dualconstellation GNSSs, in-vehicle sensors, and electronic compass. The nonlinear filter model is firstly established based on a cost-effective reduced inertial sensor system with $3 \mathrm{D}$ navigation solution. Further, the adaptive fuzzy unscented Kalman filter (AF-UKF) algorithm is developed to execute the global fusion. Specifically, a fuzzy calibration logic (FCL) is designed and introduced, which can determine the dependable degree of each received satellite measurement according to its features including current geometrical distribution and carrier-to-noise ratio. Through such mechanism, the NLOS and multipath interferences can be effectively mitigated, and accurate positioning in urban canyons can be achieved. The main novel aspect of the proposed solution is that it does not need the prior information about the driving environment and has better adaptability and accuracy.

The remainder of this paper is organized as follows. In Section 2, the filter model used for multisensor fusion is presented in detail. Then, the fusion algorithm for implementing multisensor integrated positioning is proposed in Section 3. Experimental results are provided in Section 4. Section 5 is devoted to the conclusion.

\section{Nonlinear Filter Model}

2.1. System Model. A nonlinear motion model involving the position, velocity, and attitude states based on a reduced inertial sensor system (RISS) is adopted in this paper to cut the cost. Specifically, the RISS integrates the measurements from the vertically aligned gyroscope and two horizontal accelerometers with speed readings provided by wheel speed sensors. Though reduced, it can still provide an effective 3D navigation solution for land vehicles via 3D RISS calculation mechanization [25].

2.1.1. Position and Velocity Calculation. Before describing the system equations for position and velocity, the relation between the vehicle velocity in the body frame and in the local-level frame is given by

$$
\left[\begin{array}{c}
v_{k}^{E} \\
v_{k}^{N} \\
v_{k}^{U}
\end{array}\right]=\mathbf{R}_{b, k}^{l}\left[\begin{array}{c}
0 \\
v_{k}^{w} \\
0
\end{array}\right]=\left[\begin{array}{c}
v_{k}^{w} \sin A_{k} \cos p_{k} \\
v_{k}^{w} \cos A_{k} \cos p_{k} \\
v_{k}^{w} \sin p_{k}
\end{array}\right]
$$

where

$v^{E}, v^{N}$, and $v^{U}$ denote the velocity components along east, north, and up directions, respectively.

$A, p$, and $r$ are the azimuth, pitch, and roll angles of vehicle, respectively.

$v^{w}$ is the vehicle speed derived from the vehicle's wheel speed.

$\mathbf{R}_{b, k}^{l}$ is represented as

$$
\mathbf{R}_{b, k}^{l}=\left[\begin{array}{ccc}
\cos A_{k} \cos r_{k}+\sin A_{k} \sin p_{k} \sin r_{k} & \sin A_{k} \cos p_{k} & \cos A_{k} \sin r_{k}-\sin A_{k} \sin p_{k} \cos r_{k} \\
-\sin A_{k} \cos r_{k}+\cos A_{k} \sin p_{k} \sin r_{k} & \cos A_{k} \cos p_{k} & -\sin A_{k} \sin r_{k}-\cos A_{k} \sin p_{k} \cos r_{k} \\
-\cos p_{k} \sin r_{k} & \sin p_{k} & \cos p_{k} \cos r_{k}
\end{array}\right]
$$

In (1), the velocity in the body frame is assumed to consist only of the forward longitudinal speed of the vehicle, while the transversal and vertical components are assumed to be zeros. Such constraints are very effective to improve the state estimates along the lateral and vertical directions.

Further, the latitude can be expressed as

$$
\varphi_{k}=\varphi_{k-1}+\frac{v_{k}^{N}}{R_{M}+h_{k}} \Delta t .
$$

Similarly, the longitude is

$$
\lambda_{k}=\lambda_{k-1}+\frac{v_{k}^{E}}{\left(R_{N}+h_{k}\right) \cos \varphi_{k}} \Delta t .
$$


The altitude is

$$
h_{k}=h_{k-1}+v_{k}^{U} \Delta t
$$

where

$\varphi, \lambda$, and $h$ denote the latitude, longitude, and altitude of vehicle, respectively.

$R_{M}$ is the meridian radius of curvature of the Earth.

$R_{N}$ is the normal radius of curvature of the Earth.

$\Delta t$ is the sampling time.

2.1.2. Pitch, Roll, and Azimuth Calculation. When the vehicle is moving, the forward accelerometer measures the forward vehicle acceleration as well as the component due to gravity. To calculate the pitch angle, the vehicle acceleration derived from the odometer measurements is removed from the forward accelerometer measurements:

$$
p_{k}=\sin ^{-1}\left(\frac{f_{k}^{y}-a_{k}^{w}}{g}\right) \text {. }
$$

Similarly the transversal accelerometer measures the normal component of the vehicle acceleration as well as the component due to gravity, and to calculate the roll angle, the transversal accelerometer measurement must be compensated for the normal component of acceleration:

$$
r_{k}=-\sin ^{-1}\left(\frac{f_{k}^{x}+v_{k}^{w} \omega_{k}^{z}}{g \cos p_{k}}\right) .
$$

Besides, the azimuth angle directly in local-level frame can be written as

$$
\begin{aligned}
A_{k}= & A_{k-1}-\omega_{k}^{z} \Delta t+\left(\omega^{e} \sin \varphi_{k-1}\right) \Delta t \\
& +\frac{v_{k-1}^{E} \tan \varphi_{k-1}}{\left(R_{N}+h_{k-1}\right)} \Delta t,
\end{aligned}
$$

where

$a^{w}$ is the vehicle acceleration derived from the vehicle's wheel speed.

$f^{x}$ and $f^{y}$ devote the transversal accelerometer measurement and forward accelerometer measurement, respectively.

$\omega^{z}$ is the angular rate obtained from the vertically aligned gyroscope.

$\omega^{e}$ is the Earth's rotation rate.

$g$ is the acceleration of gravity.

2.1.3. Stochastic Errors. In order to compensate for the stochastic errors associated with the gyroscope and the vehicle's wheel speed and enhance the estimation accuracy of the filter, these stochastic errors are also included as states, which are modeled by a Gauss-Markov model:

$$
\left[\begin{array}{l}
\delta F_{k}^{w} \\
\delta \omega_{k}^{z}
\end{array}\right]=\left[\begin{array}{l}
\left(1-\gamma_{w} \Delta t\right) \delta F_{k-1}^{w} \\
\left(1-\beta_{z} \Delta t\right) \delta \omega_{k-1}^{z}
\end{array}\right]+\left[\begin{array}{c}
\sqrt{2 \gamma_{w} \sigma_{w}^{2} \Delta t} \\
\sqrt{2 \beta_{z} \sigma_{z}^{2} \Delta t}
\end{array}\right],
$$

where $\delta F^{w}$ is the scale factor error of the wheel speed.

$\delta \omega^{Z}$ is the stochastic gyroscope drift.

$\gamma_{w}$ is the reciprocal of the autocorrelation time for the scale factor of the wheel speed.

$\beta_{z}$ is the reciprocal of the autocorrelation time for the gyroscope's stochastic drift.

$\sigma_{w}^{2}$ is the variance of the noise associated with the wheel speed.

$\sigma_{z}^{2}$ is the variance of the noise associated with the gyroscope's stochastic drift.

Based on 3D RISS as described by (1)-(9), the nonlinear system transition model about vehicle states is given by

$\mathbf{X}_{k}^{\mathrm{RISS}}=\left[\begin{array}{c}\varphi_{k} \\ \lambda_{k} \\ h_{k} \\ v_{k}^{E} \\ v_{k}^{N} \\ v_{k}^{U} \\ p_{k} \\ r_{k} \\ A_{k} \\ \delta F_{k}^{w} \\ \delta \omega_{k}^{Z}\end{array}\right]$

$$
\left[\begin{array}{c}
\varphi_{k-1}+\frac{v_{k}^{N}}{R_{M}+h_{k}} \Delta t \\
\lambda_{k-1}+\frac{v_{k}^{E}}{\left(R_{N}+h_{k}\right) \cos \varphi_{k}} \Delta t \\
h_{k-1}+v_{k}^{U} \Delta t \\
v_{k}^{w} \sin A_{k} \cos p_{k} \\
v_{k}^{w} \cos A_{k} \cos p_{k} \\
v_{k}^{w} \sin p_{k} \\
\sin ^{-1}\left(\frac{f_{k}^{y}-a_{k}^{w}}{g}\right) \\
-\sin ^{-1}\left(\frac{f_{k}^{x}+v_{k}^{w} \omega_{k}^{z}}{g \cos p_{k}}\right) \\
A_{k-1}-\omega_{k}^{z} \Delta t+\left(\omega^{e} \sin \varphi_{k-1}\right) \Delta t+\frac{v_{k-1}^{E} \tan \varphi_{k-1}}{\left(R_{N}+h_{k-1}\right)} \Delta t \\
\left(1-\gamma_{w} \Delta t\right) \delta F_{k-1}^{w} \\
\left(1-\beta_{z} \Delta t\right) \delta \omega_{k-1}^{Z}
\end{array}\right]
$$


Moreover, the receiver's clock bias and drift errors of GPS and BeiDou can be modeled as

$$
\begin{aligned}
& \mathbf{X}_{k}^{\mathrm{GPS}}=\left[\begin{array}{c}
\delta b_{k}^{\mathrm{GPS}} \\
\delta d_{k}^{\mathrm{GPS}}
\end{array}\right]=\left[\begin{array}{c}
\delta b_{k-1}^{\mathrm{GPS}}+\delta d_{k-1}^{\mathrm{GPS}} \Delta t \\
\delta d_{k-1}^{\mathrm{GPS}}
\end{array}\right], \\
& \mathbf{X}_{k}^{\mathrm{BDS}}=\left[\begin{array}{c}
\delta b_{k}^{\mathrm{BDS}} \\
\delta d_{k}^{\mathrm{BDS}}
\end{array}\right]=\left[\begin{array}{c}
\delta b_{k-1}^{\mathrm{BDS}}+\delta d_{k-1}^{\mathrm{BDS}} \Delta t \\
\delta d_{k-1}^{\mathrm{BDS}}
\end{array}\right],
\end{aligned}
$$

where $\delta b^{\mathrm{GPS}}$ and $\delta d^{\mathrm{GPS}}$ are GPS receiver's clock offset and drift error, respectively. $\delta b^{\mathrm{BDS}}$ and $\delta d^{\mathrm{BDS}}$ are BeiDou receiver's clock offset and drift error, respectively.
Combining (10) and (11), we can obtain the full system state transition model for the overall tightly coupled integration as

$$
\mathbf{X}(k)=\mathbf{f}(\mathbf{X}(k-1), \mathbf{U}(k-1), \mathbf{W}(k-1), \mathbf{T}(k-1)),
$$

where $\mathbf{X}$ and $\mathbf{U}$ represent the state and input vectors of the system, respectively, and $\mathbf{W}$ and $\mathbf{T}$ are the corresponding system and input noise vectors, respectively. $\mathbf{f}(\cdot)$ is the nonlinear system function where the function components can be determined based on (10) and (11). $\mathbf{X}$ and $\mathbf{U}$ can be represented by

$$
\begin{aligned}
& \mathbf{X}=\left[\begin{array}{llllllllllllllll}
\varphi & \lambda & h & v^{E} & v^{N} & v^{U} & p & r & A & \delta F^{w} & \delta \omega^{z} & \delta b^{\mathrm{GPS}} & \delta d^{\mathrm{GPS}} & \delta b^{\mathrm{BDS}} & \delta d^{\mathrm{BDS}}
\end{array}\right]^{T}, \\
& \mathbf{U}=\left[\begin{array}{lllll}
v^{w} & a^{w} & f^{x} & f^{y} & \omega^{z}
\end{array}\right]^{T} .
\end{aligned}
$$

2.2. Measurement Model. In the tightly coupled integration, the pseudorange measurements of dual-constellation GNSSs act as the observation vector. Therefore, the measurement model can be given as

$$
\mathbf{Z}(k)=\mathbf{h}(\mathbf{X}(k))+\boldsymbol{\varepsilon}(k),
$$

where $\mathbf{Z}$ is the measurement vector, $\mathbf{h}(\cdot)$ is the process function of the measurement model, and $\boldsymbol{\varepsilon}$ is the measurement Gaussian noise vector. $\mathbf{Z}$ can be depicted as

$$
\begin{aligned}
& \text { Z }
\end{aligned}
$$

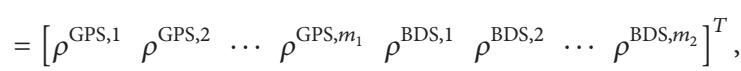

where $\rho^{\text {GPS }}$ and $\rho^{\text {BDS }}$ are the corrected GPS and BeiDou pseudorange measurements, respectively. $m_{1}$ and $m_{2}$ are the numbers of received GPS and BeiDou satellites whose elevation angle and carrier-to-noise ratio are both more than certain thresholds (e.g., $35^{\circ}$ and $30 \mathrm{db}-\mathrm{Hz}$, resp., in this paper), respectively. In urban areas, through such threshold processing, that is, abandoning the measurements of the satellites with low elevation angle and low carrier-to-noise ratio, the impact of NLOS and multipath can be preliminarily alleviated to some degree.

Note that in common INS/GNSS tightly coupled integration, each of received satellite measurements is generally regarded as Gaussian noise with the same noise covariance
$R_{0}$. That is, $\boldsymbol{\varepsilon}$ is usually assumed to have the noise covariance matrix $\mathbf{R}$, which has equal diagonal elements; that is,

$$
\mathbf{R}(k)=\operatorname{diag}\left[\begin{array}{lll}
R_{0} & \cdots & R_{0}
\end{array}\right],
$$

where $\mathbf{R}$ has $m_{1}+m_{2}$ dimension.

Satellite clock bias and ionospheric errors can be calculated from the satellite's navigation message, and tropospheric error can also be estimated using an appropriate model [25]. Hence, after correcting all the errors except receiver clock errors, the corrected pseudoranges $\rho^{\mathrm{GPS}}$ and $\rho^{\mathrm{BDS}}$ can be described as

$$
\begin{aligned}
& \rho^{\mathrm{GPS}}=\left\|\mathbf{r}-\mathbf{r}^{\mathrm{GPS}}\right\|+\delta b^{\mathrm{GPS}}+\varepsilon^{\mathrm{GPS}, \rho}, \\
& \rho^{\mathrm{BDS}}=\left\|\mathbf{r}-\mathbf{r}^{\mathrm{BDS}}\right\|+\delta b^{\mathrm{BDS}}+\varepsilon^{\mathrm{BDS}, \rho},
\end{aligned}
$$

where $\mathbf{r}=\left[\begin{array}{lll}x & y & z\end{array}\right]$ is the receiver's rectangular coordi-

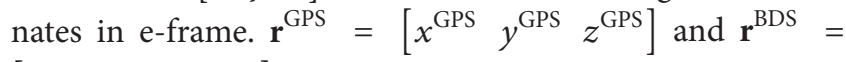
$\left[x^{\mathrm{BDS}} y^{\mathrm{BDS}} z^{\mathrm{BDS}}\right]$ are satellites' rectangular coordinates in e-frame. $\delta b^{\mathrm{GPS}}$ and $\delta b^{\mathrm{BDS}}$ are the GPS and BeiDou receiver's clock biases, respectively. $\varepsilon^{\mathrm{GPS}, \rho}$ and $\varepsilon^{\mathrm{BDS}, \rho}$ are the total effect of residual errors.

The coordinate transformation between geodetic e-frame and rectangular e-frame is

$$
\begin{aligned}
& x=\left(R_{N}+h\right) \cos \varphi \cos \lambda, \\
& y=\left(R_{N}+h\right) \cos \varphi \sin \lambda, \\
& z=\left[R_{N}\left(1-e^{2}\right)+h\right] \sin \varphi,
\end{aligned}
$$

where $e$ is the eccentricity of ellipsoid. Utilizing this transformation, the measurement equation can be obtained as 
$\mathbf{Z}(k)$

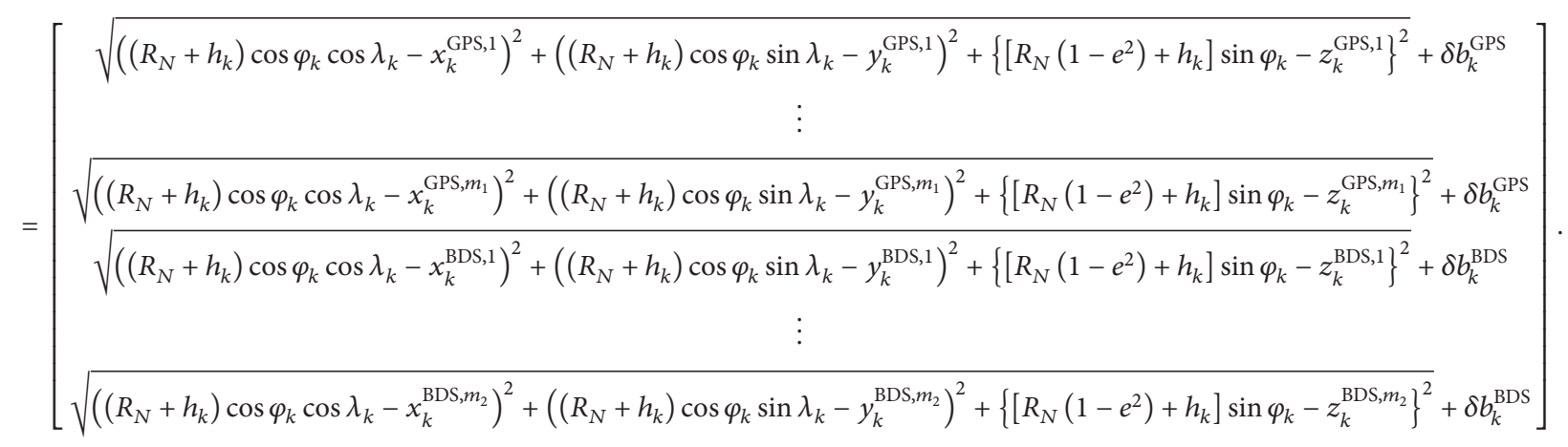

\section{Fusion Positioning Algorithm}

3.1. EKF Algorithm. The extended Kalman filter (EKF) has been widely used for INS/GNSS integration. For system state model (12) and measurement model (19), the system, input, and measurement noises are assumed to be Gaussian with zero mean and their covariance matrices $\mathbf{Q}, \boldsymbol{\Gamma}$, and $\mathbf{R}$, respectively. Further, the corresponding EKF process can be divided into the following two phases:

Prediction

$$
\begin{aligned}
\widehat{\mathbf{X}}(k, k-1)= & \mathbf{f}(\mathbf{X}(k-1), \mathbf{U}(k-1), \mathbf{0}, \mathbf{0}), \\
\mathbf{P}(k, k-1)= & \mathbf{A}(k, k-1) \mathbf{P}(k-1) \mathbf{A}^{T}(k, k-1) \\
& +\mathbf{B}(k, k-1) \mathbf{\Gamma}(k-1) \mathbf{B}^{T}(k, k-1) \\
& +\mathbf{Q}(k-1) .
\end{aligned}
$$

Update

$$
\begin{aligned}
\mathbf{K}(k)= & \mathbf{P}(k, k-1) \cdot \mathbf{H}^{T}(k) \\
& \cdot\left[\mathbf{H}(k) \mathbf{P}(k, k-1) \mathbf{H}^{T}(k)+\mathbf{R}(k)\right]^{-1}, \\
\widehat{\mathbf{X}}(k)=\widehat{\mathbf{X}}(k, k-1) & \\
& +\mathbf{K}(k)[\mathbf{Z}(k)-\mathbf{h}(\widehat{\mathbf{X}}(k, k-1))], \\
\mathbf{P}(k)= & {[\mathbf{I}-\mathbf{K}(k) \cdot \mathbf{H}(k)] \cdot \mathbf{P}(k, k-1), }
\end{aligned}
$$

where $\mathbf{I}$ is an identity matrix, $\mathbf{A}$ and $\mathbf{B}$ are the Jacobian matrices of the system function $\mathbf{f}(\cdot)$ with respect to $\mathbf{X}$ and $\mathbf{U}$, and $\mathbf{H}$ is the Jacobian matrix of the measurement function $\mathbf{h}(\cdot)$ with respect to $\mathbf{X}$; that is,

$$
\begin{aligned}
\mathbf{A}_{[i][j]} & =\frac{\partial \mathbf{f}_{[i]}}{\partial x_{[j]}}(\widehat{\mathbf{X}}(k, k-1), \mathbf{U}(k-1), \mathbf{0}, \mathbf{0}), \\
\mathbf{B}_{[i][j]} & =\frac{\partial \mathbf{f}_{[i]}}{\partial u_{[j]}}(\widehat{\mathbf{X}}(k, k-1), \mathbf{U}(k-1), \mathbf{0}, \mathbf{0}),
\end{aligned}
$$

$$
\mathbf{H}_{[i][j]}=\frac{\partial \mathbf{h}_{[i]}}{\partial x_{[j]}}(\widehat{\mathbf{X}}(k, k-1)) .
$$

3.2. UKF Algorithm. As shown in (12) and (19), both system and measurement models are nonlinear. Although the EKF can deal with such fusion through linearization, it will inevitably cause the approximation error. To enhance the integration performance, nonlinear estimation techniques that do not require linearized dynamic models should be considered to be used. The unscented Kalman filter (UKF) is one of the effective nonlinear Bayes filters which can directly use nonlinear system and measurement models without any linearization [26, 27]. Generally, it can achieve a good balance between computational complexity and accuracy [28]. In contrast to the EKF, the UKF does not need to compute the Jacobian matrix and can predict the means and covariance correctly up to the fourth order.

For (12) and (19), we can execute the recursive procedure of UKF according to the following steps [26, 27]:

Step 1. Calculate weights coefficient and sigma points:

$$
\begin{aligned}
\omega_{0}^{(m)} & =\frac{\eta}{n+\eta}, \quad i=0, \\
\omega_{0}^{(c)} & =\frac{\eta}{n+\eta}+\left(1-\alpha_{1}^{2}+\alpha_{2}\right), \quad i=0, \\
\omega_{i}^{(m)} & =\omega_{i}^{(m)}=\frac{1}{2(n+\eta)}, \quad i=1,2, \ldots, 2 n, \\
\xi_{i}(k-1) & =\overline{\mathbf{X}}(k-1), \quad i=0, \\
\xi_{i}(k-1) & =\overline{\mathbf{X}}(k-1)+(\sqrt{(n+\eta) \mathbf{P}(k)})_{i}, \\
\xi_{i}(k-1) & =\overline{\mathbf{X}}(k-1)-(\sqrt{(n+\eta) \mathbf{P}(k)})_{i}, \\
i=n+1, n+2, \ldots, 2 n, &
\end{aligned}
$$


where $\eta=\alpha_{1}{ }^{2}\left(n+\alpha_{3}\right)-n$ is a scaling parameter. The constant $\alpha_{1}$ determines the spread of the sigma points around a mean of state $\mathbf{X}$ and is usually set to a small positive value. The constant $\alpha_{3}$ is usually set to $0 . \alpha_{2}$ is used to incorporate prior knowledge of the distribution of $\mathbf{X}$ and is optimally set to 2 for Gaussian distributions; $n$ is the dimension of state vector.

Step 2. Estimate a priori state $\widehat{\mathbf{X}}(k, k-1)$ and a priori error covariance $\mathbf{P}(k, k-1)$ and then obtain the predicted observation by the unscented transform of which inputs are the sigma points and the weights:

$$
\begin{aligned}
\boldsymbol{\xi}_{i}(k, k-1)= & \mathbf{f}\left(\boldsymbol{\xi}_{i}(k-1)\right), \quad i=0,1, \ldots, 2 n, \\
\widehat{\mathbf{X}}(k, k-1)= & \sum_{i=0}^{2 n} \omega_{i}^{(m)} \boldsymbol{\xi}_{i}(k, k-1), \\
\mathbf{P}(k, k-1)= & \mathbf{Q}(k) \\
& +\sum_{i=0}^{2 n} \omega_{i}^{(c)}\left[\boldsymbol{\xi}_{i}(k, k-1)-\widehat{\mathbf{X}}(k, k-1)\right] \\
& \cdot\left[\boldsymbol{\xi}_{i}(k, k-1)-\widehat{\mathbf{X}}(k, k-1)\right]^{T}, \\
\boldsymbol{\zeta}_{i}(k, k-1)= & \mathbf{h}\left(\boldsymbol{\xi}_{i}(k, k-1)\right), \quad i=0,1, \ldots, 2 n, \\
\widehat{\mathbf{Z}}(k, k-1)= & \sum_{i=0}^{2 n} \omega_{i}^{(m)} \boldsymbol{\zeta}_{i}(k, k-1) .
\end{aligned}
$$

Step 3. Calculate the UKF gain:

$$
\begin{aligned}
& \mathbf{P}_{Z Z}= \mathbf{R}(k)+\sum_{i=0}^{2 n} \omega_{i}^{(c)}\left[\zeta_{i}(k, k-1)-\widehat{\mathbf{Z}}(k, k-1)\right] \\
& \cdot\left[\zeta_{i}(k, k-1)-\widehat{\mathbf{Z}}(k, k-1)\right]^{T}, \\
& \mathbf{P}_{X Z}=\sum_{i=0}^{2 n} \omega_{i}^{(c)}\left[\xi_{i}(k, k-1)-\widehat{\mathbf{X}}(k, k-1)\right] \\
& \cdot\left[\zeta_{i}(k, k-1)-\widehat{\mathbf{Z}}(k, k-1)\right]^{T}, \\
& \mathbf{K}(k)=\mathbf{P}_{X Z} \mathbf{P}_{Z Z}{ }^{-1} .
\end{aligned}
$$

Step 4. Update the system state and error covariance:

$$
\begin{aligned}
\widehat{\mathbf{X}}(k) & =\widehat{\mathbf{X}}(k, k-1)+\mathbf{K}(k)(\mathbf{Z}(k)-\widehat{\mathbf{Z}}(k, k-1)), \\
\mathbf{P}(k) & =\mathbf{P}(k, k-1)-\mathbf{K}(k) \mathbf{P}_{Z Z} \mathbf{K}(k)^{T} .
\end{aligned}
$$

3.3. AF-UKF Algorithm. For the UKF above, the noise covariance matrix $\mathbf{R}(k)$ in (25) is usually set to be a constant diagonal one. This setting is generally appropriate in the open-sky situations but is not suitable for urban canyons. As mentioned earlier, the satellite signals in urban areas are often blocked and/or reflected by surrounding objects causing NLOS and multipath interferences. For the received LOS satellites, their measurement accuracy is superior. However, if one received satellite signal belongs to multipath and
NLOS, its measurement accuracy is poor. Through simple threshold processing mentioned above, only partly obvious NLOS and multipath interferences can be alleviated. That is, such alleviation is very limited and rough. For the remaining satellite signals, it is still necessary to adaptively adjust the dependence on each satellite measurement whether from BeiDou or from GPS according to its specific conditions rather than utilize it invariably.

Generally, in unban canyon scenarios, the LOS satellite signals may be different from the satellite signals affected by the NLOS and multipath interferences in several aspects. First, the satellite signals along the vehicle's longitudinal direction (i.e., along the street) are not obstructed by buildings and trees, while the signals going along the vehicle's lateral direction (i.e., across the street) are prone to be blocked or reflected, as illustrated in Figure 1. Besides, the satellite signals with low elevation angles are more likely to be influenced than those with high elevation angles in urban canyons. Moreover, the LOS signals often have higher carrierto-noise ratio (i.e., $C / N_{0}$ ) than the NLOS and multipath signals [29].

Obviously, if we can take full advantages of the abovementioned features to determine the degree of dependence on the measurement of each received satellite signal, the impact of NLOS and multipath interferences can be mitigated and thus the positioning accuracy can be enhanced. However, note that how to utilize the features above to ascertain the dependence degree on the measurement of each received satellite signal is a nonlinear process with some uncertainty and fuzziness, rather than a strict or rigorous process. Thus, it is reasonable to adopt fuzzy logic to model this decision process that is based on the features of received satellite signals.

Based on the discussions above, the fuzzy calibration logic (FCL) here is designed using three inputs, that is, the azimuth difference $\theta_{i}$ between the received $i$ th satellite and the vehicle, the elevation angle $\gamma_{i}$ of the received $i$ th satellite, and the carrier-to-noise ratio $P_{i}$ (i.e., $C / N_{0}$ ) and one output, the scaling coefficient $\mu_{i}$, which is used to adjust the pseudorange measurement noise level. In actual implementation, $\theta$ is determined by

$$
\theta_{i}=\left|A^{S_{i}}-A^{C}\right|, \quad\left(i=1, \ldots, m_{1}+m_{2}\right),
$$

where $A^{S_{i}}$ denotes the azimuth angle of the received $i$ th satellite and $A^{C}$ is the azimuth angle measured by the electronic compass. If $\theta_{i}$ is small, it means that the signal of received $i$ th satellite is nearly along the canyon's direction and its observation noise covariance should not be enlarged. On the other hand, large $\theta_{i}$ means that the signal of received $i$ th satellite significantly deviates the canyon's direction and its observation noise covariance should be enlarged. $\mu_{i}$ represents the enlarged degree about the observation noise covariance of the received $i$ th satellite; that is,

$$
R_{i}^{2}=\left(\mu_{i} \times R_{0}\right)^{2}, \quad\left(i=1, \ldots, m_{1}+m_{2}\right) .
$$

The range of $\mu_{i}$ is set mainly according to the knowledge or experience about the observation noise and then is adjusted 


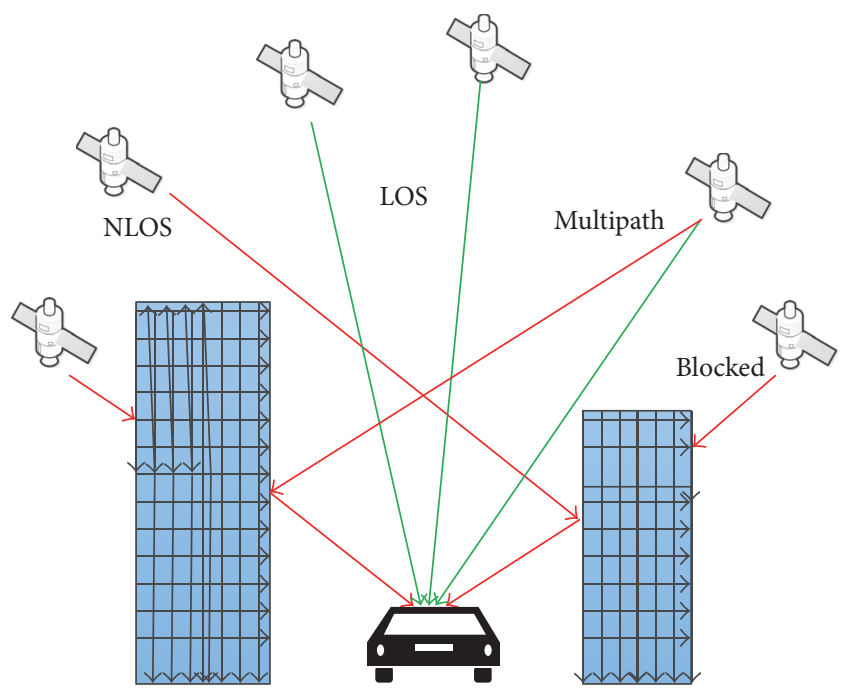

(a)

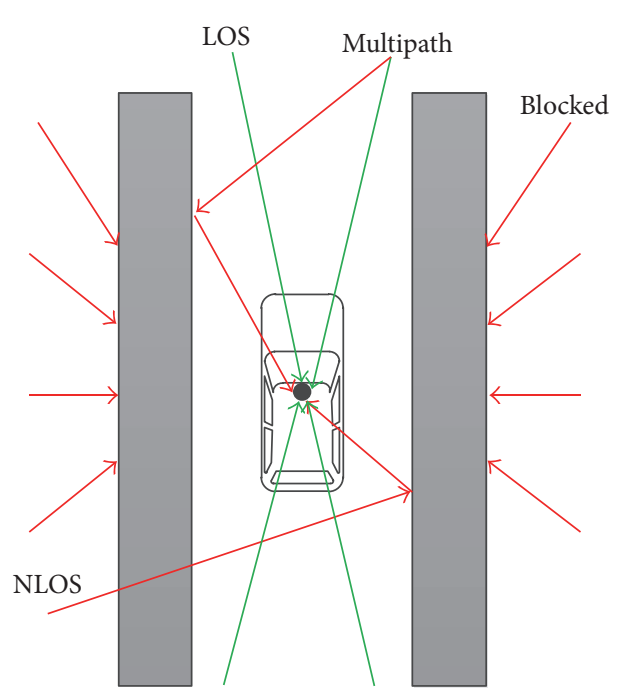

(b)

FIGURE 1: Illustration of NLOS and multipath interferences in urban canyons. (a) Front view. (b) Top view.

many times by trial and error to obtain better estimation performance.

Three membership functions, Large (L), Middle (M), and Small (S), and three membership functions, Low Elevation (LowE), Middle Elevation (MidE), and High Elevation (HighE), are used to describe two input variables, $\theta_{i}$ and $\gamma_{i}$, respectively. Besides, two membership functions, Low Power (LowP) and High Power (HighP), are utilized to describe the input variable $P_{i}$. For the output, six membership functions are defined as Normal (N-A), Slight Amplification (SL-A), Small Amplification (SM-A), Middle Amplification (M-A), Large Amplification (L-A), and Very Large Amplification (VL-A). The fuzzy membership functions for three inputs and one output are shown in Figure 2.

The fuzzy reasoning rules are mainly based on the following prior considerations. First, if $\theta_{i}$ is Small, $\gamma_{i}$ is High Elevation, and $P_{i}$ is High Power, then it means that the received $i$ th satellite is not interfered and its observation noise covariance should not be enlarged (i.e., Normal). Besides, if $\theta_{i}$ is Large and $\gamma_{i}$ is Low Elevation, it reveals that the received $i$ th satellite is very likely to be affected by urban canyons and thus its observation noise covariance should be amplified largely or very largely. Finally, in the cases of other input combinations, the observation noise covariance should be enlarged moderately. Table 1 gives the complete fuzzy rule base.

When the enlarged degree about the observation noise covariance of each received satellite is determined utilizing the FCL above, we can achieve the global fusion via the UKF algorithm presented in Section 3.2. For clarity, we summarize the proposed adaptive fuzzy UKF (AF-UKF) algorithm briefly as follows:

Step 1. Calculate weights coefficient and sigma points according to (23).
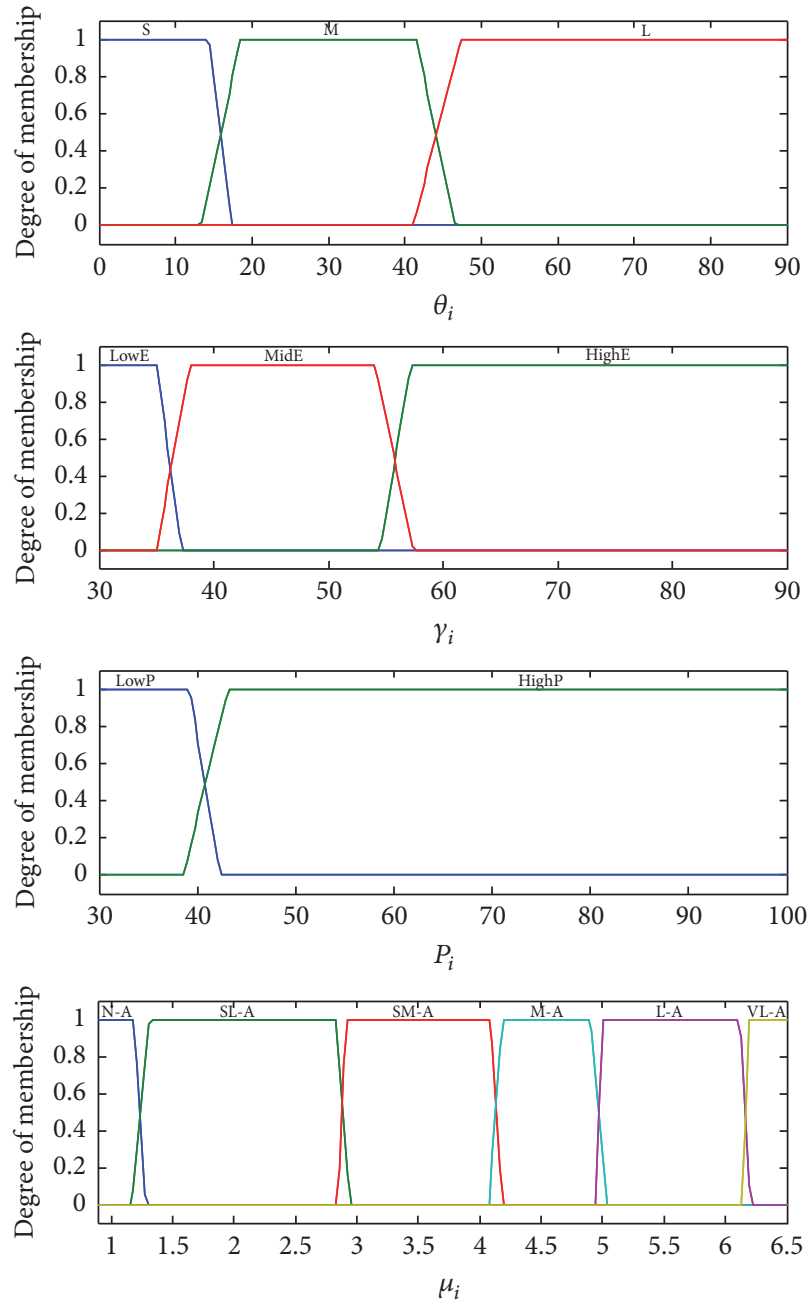

Figure 2: Membership functions for $\theta_{i}, \gamma_{i}$, and $P_{i}$. 
TABLE 1: Fuzzy rules for proposed FCL.

\begin{tabular}{ccccc}
\hline$\theta_{i}$ & $P_{i}$ & HighE & $\begin{array}{c}\gamma_{i} \\
\text { MidE }\end{array}$ & LowE \\
\hline \multirow{2}{*}{ S } & HighP & N-A & SL-A & SL-A \\
& LowP & N-A & SM-A & SM-A \\
\multirow{2}{*}{ M } & HighP & N-A & SM-A & M-A \\
& LowP & SM-A & L-A & L-A \\
\multirow{2}{*}{ L } & HighP & SL-A & M-A & L-A \\
& LowP & SM-A & L-A & VL-A \\
\hline
\end{tabular}

Step 2. Estimate a priori state and its error covariance and then calculate the predict observation based on (24).

Step 3. For each received satellite signal, its azimuth difference $\theta_{i}$ is ascertained according to (29) and then the corresponding scaling coefficient $\mu_{i}$ can be determined utilizing the FCL above. When all satellite signals are judged, the whole adaptive noise covariance matrix $\mathbf{R}_{\mathbf{a}}$ can be obtained:

$$
\begin{aligned}
& \mathbf{R}_{\mathbf{a}}(k) \\
& \quad=\operatorname{diag}\left[\begin{array}{lllll}
\left(\mu_{1} R_{0}\right)^{2} & \cdots & \left(\mu_{i} R_{0}\right)^{2} & \cdots & \left(\mu_{m_{1}+m_{2}} R_{0}\right)^{2}
\end{array}\right] .
\end{aligned}
$$

Step 4. Substitute $\mathbf{R}$ in (25) with $\mathbf{R}_{\mathbf{a}}$ and then calculate the UKF gain according to (25)-(27).

Step 5. Update the system state and error covariance based on (28).

From the discussion above, the established FCL is mainly based on the information about the urban environment knowledge and received satellite signal features.

\section{Experimental Results}

4.1. Experiment Setup. To verify the positioning performance of the proposed solution, experiments were conducted on a Chery TIGGO5 SUV vehicle. The information about wheel speeds could be directly obtained from the in-vehicle CAN network. Besides, a low-cost NovAtel C260-AT GPS/BeiDou dual-constellation receiver, MEMSIC MEMS-based IMU VG440CA-200 inertial sensors, and KVH C100plus electronic compass were installed. The sensor accuracies $(1 \sigma)$ are $5 \mathrm{~m}$ for GPS and BeiDou pseudorange measurements, $0.1 \mathrm{~m} / \mathrm{s}^{2}$ for the accelerometers, $0.2^{\circ} / \mathrm{s}$ for the yaw rate sensor, $0.5^{\circ}$ for the yaw angle of electronic compass, and $0.05 \mathrm{~m} / \mathrm{s}$ for wheel speed sensors. Moreover, an accurate and reliable NovAtel SPAN-CPT system was used as a reference, which can provide accurate positioning reference via postprocessing even under adverse environments [30].

The experiments were executed along a typical urbancanyon trajectory in the downtown of Nanjing City, which is a metropolitan city in East China and has a population of over 8 million. Figure 3 shows this trajectory. There are many tall buildings and skyscrapers along the two sides of the test trajectory. The same FCL calibration parameters are utilized throughout the whole experiment.

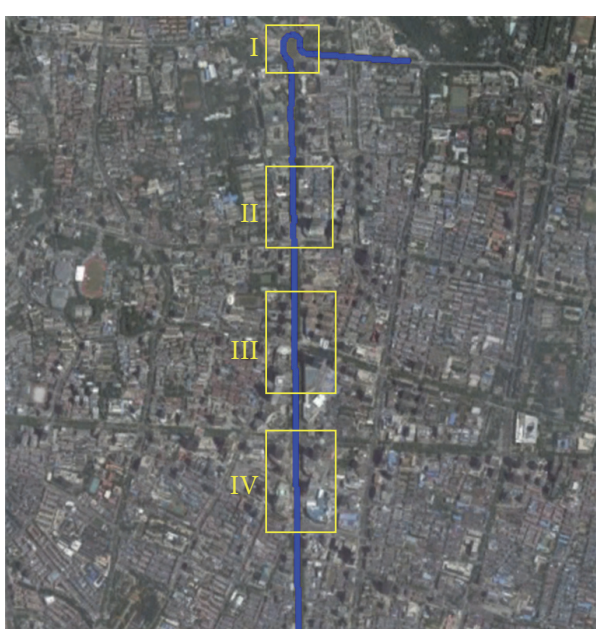

FIGURE 3: Test trajectory for the performance evaluation (Courtesy of Google Earth).

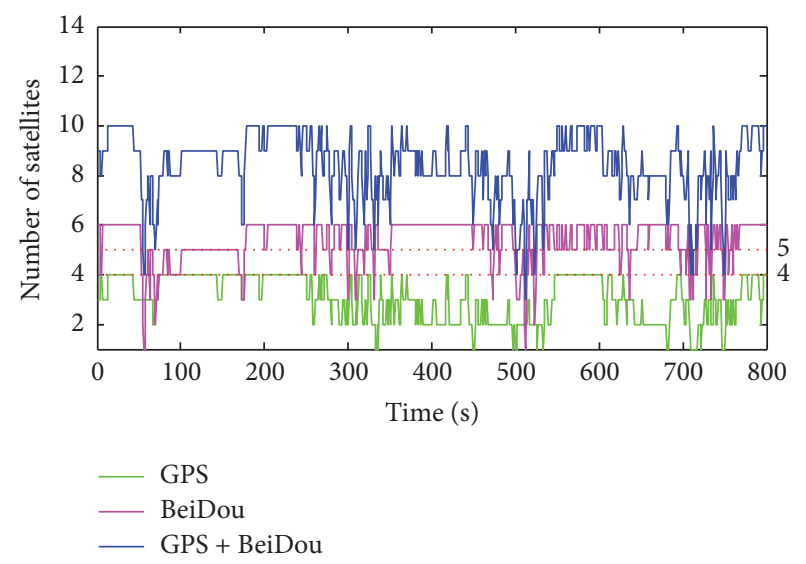

FIGURE 4: Number of received satellites.

4.2. Satellite Availability Evaluation. Figure 4 shows the number of received satellites whose elevation angle and carrierto-noise ratio are more than $35^{\circ}$ and $30 \mathrm{db}-\mathrm{Hz}$, respectively. As shown in Figure 4, it is obvious that with more satellite constellations utilized, more satellites can be available in city canyons.

With standalone GPS used, the number of available satellites is less than four at over $50.1 \%$ epochs, which is not sufficient to provide a positioning solution. With only BeiDou utilized, the percentage of the situation when the number of available satellites is less than four can be reduced to $5.2 \%$. In contrast, with the combination of GPS and BeiDou, the satellite availability can be obviously enhanced. The percentage of the situation when the number of available satellites is insufficient to provide a positioning solution (i.e., $<5)$ can be decreased to only $1.2 \%$. These results show the multiconstellation GNSSs have the potential to improve the positioning performance in challenging urban environments. However, note that the multiconstellation GNSSs can still not ensure providing $100 \%$ positioning solution in urban canons. 
TABLE 2: Statistics of Euclidean distance errors in four regions (unit: $\mathrm{m}$ ).

\begin{tabular}{lcccccrrr}
\hline \multirow{2}{*}{ Region number } & \multicolumn{2}{c}{ EKF } & \multicolumn{2}{c}{ UKF } & \multicolumn{2}{c}{ AF-EKF } & \multicolumn{2}{c}{ AF-UKF } \\
& Max. & RMS & Max. & RMS & Max. & RMS & Max. & RMS \\
\hline I & 8.47 & 3.61 & 8.15 & 3.42 & 6.28 & 3.22 & 5.31 \\
II & 36.9 & 13.09 & 29.34 & 9.96 & 10.81 & 4.89 & 9.72 & 4.62 \\
III & 21.87 & 6.05 & 22.54 & 6.21 & 10.11 & 4.54 & 10.04 & 4.59 \\
IV & 60.68 & 12.85 & 46.92 & 10.73 & 11.97 & 4.73 & 11.52 \\
\hline
\end{tabular}

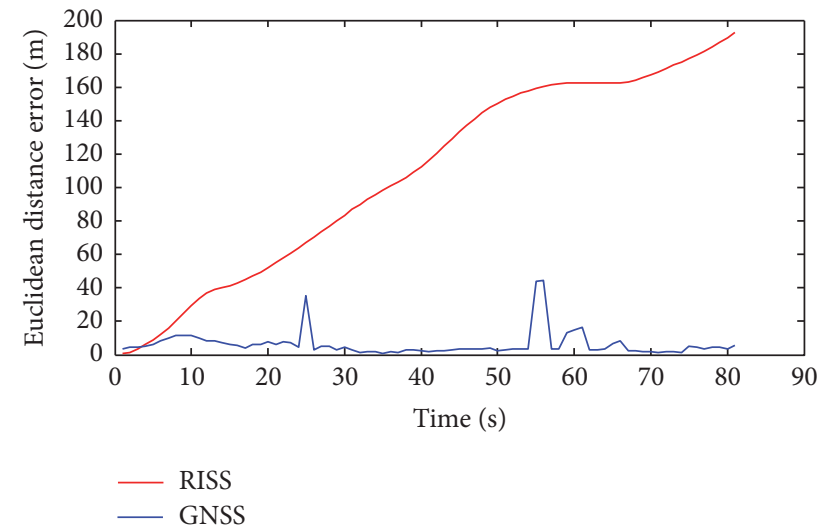

FIGURE 5: Euclidean distance errors of GNSS only and RISS only in Region IV.

4.3. Positioning Performance Evaluation. Firstly, we evaluate the performance of using GNSS only and RISS only. The positioning result in Region IV, shown in Figure 5, is selected as an example to demonstrate the performance of GNSS only and RISS only due to the fact that similar results can be obtained from other regions. For the RISS only, its initial position is set to the reference position at the start of Region IV. The Euclidean distance error (horizontal positioning errors) of RISS only grows with time and reaches $190 \mathrm{~m}$ in $80 \mathrm{~s}$ GNSS outage, which can be attributed to the accumulation of inertial sensor errors. The performance of GNSS only is better than the RISS only, but the maximum error of GNSS only is up to $44.5 \mathrm{~m}$ owing to the NLOS and multipath interferences. From this, we can find that the performances of using GNSS only or RISS only are so poor that they cannot satisfy the requirements of vehicle positioning in urban canyons. Therefore, we focus on the evaluation of positioning performance fusing GNSS and other low-cost complementary sensors in the following sections.

The positioning performance of the proposed AF-UKF solution is assessed through comparing with three other representative positioning methods, that is, EKF and UKF presented in Sections 3.1 and 3.2 and AF-EKF. Note that four typical driving regions where dense tall buildings and skyscrapers are located nearby, that is, Regions I-IV in Figure 3 , are selected to make the statistical analysis.

For four positioning methods, Table 2 gives their statistics of Euclidean distance errors (horizontal positioning errors) in four selected regions. To provide a clear description, Figures 6 and 7 illustrate the positioning results of three methods (i.e., EKF, UKF, and AF-UKF) on the map in Regions I and IV,

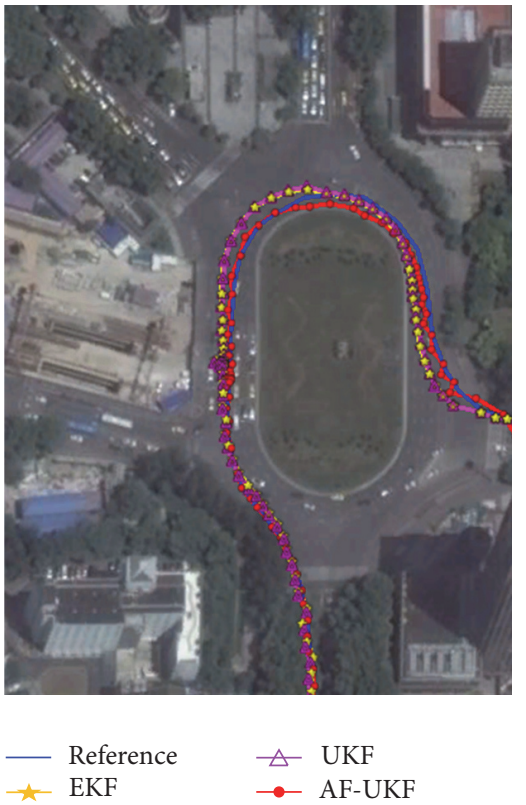

FIGURE 6: Positioning results of three methods on the map in Region I.

respectively. For brevity, the results in two other regions are not shown.

From the results above, all three fusion positioning methods can obtain $100 \%$ positioning solution during the test, which can overcome the disadvantage of the positioning method of multiconstellation GNSSs alone discussed in Section 4.2.

As far as the EKF and UKF are concerned, the UKF exhibits better nonlinear adaptability and can achieve better performance than the EKF in most cases. For instance, in Region I, the maximum and RMS values of Euclidean distance error of EKF is $8.47 \mathrm{~m}$ and $3.61 \mathrm{~m}$, respectively; whereas those of UKF are decreased to $8.15 \mathrm{~m}$ and $3.42 \mathrm{~m}$, respectively. However, in the situation where there exist obvious NLOS and multipath interferences (e.g., in Regions II-IV), their adverse effect cannot be effectively mitigated by both of the EKF and UKF methods. As illustrated in Regions II-IV, whether for EKF or for UKF, the corresponding maximum values of Euclidean distance error are large.

Compared with the EKF and UKF methods, both the AFEKF and AF-UKF can significantly enhance the positioning performance. However, the proposed AF-UKF is able to obtain better performance than AF-EKF in most cases. From Table 2, in all regions, the AF-UKF solution can achieve the 


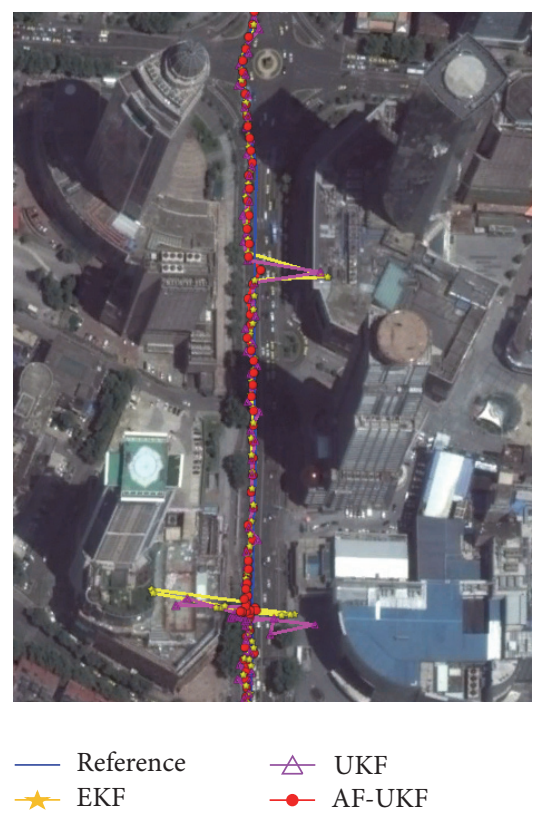

FIGURE 7: Positioning results of three methods on the map in Region IV.

best positioning accuracy. Specifically, even in the environments of heavy NLOS and multipath interferences, it can still provide reasonable and acceptable accurate positioning for common positioning applications. For example, in Region IV where there are dense tall buildings and skyscrapers nearby as shown in Figure 7, the maximum values of Euclidean distance error of EKF and UKF reach $60.68 \mathrm{~m}$ and $46.92 \mathrm{~m}$, respectively. In contrast, the value of AF-UKF is only $11.52 \mathrm{~m}$, that is, the significant improvement of about $81 \%$ and $75 \%$ over EKF and UKF, respectively. It can be attributed to the fact that the proposed fuzzy calibration logic in AF-UKF can effectively alleviate the impact of NLOS and multipath propagations.

\section{Conclusion}

In this paper, we propose a tightly coupled positioning solution for land vehicles based on the adaptive fuzzy unscented Kalman filter (AF-UKF) integrating dualconstellation GNSSs with other low-cost complementary sensors.

The nonlinear filter model is first built based on a costeffective reduced inertial sensor system with 3D navigation solution. Then, the adaptive fuzzy unscented Kalman filter algorithm is proposed, which can effectively mitigate the NLOS and multipath interferences and achieve accurate positioning in urban canyons. Finally, the proposed solution is validated through experiments in real urban canyons. The main advantage of the proposed solution is that it does not need the accurate prior information about the driving environment and owns superior adaptability and accuracy.

It should be noted that the proposed solution also has its own limitation. Under certain conditions, the observation noise covariance of some satellites, which seem suspicious but are actually healthy, would be amplified largely or very largely and may cause the loss in the positioning accuracy to some extent. If 3D city map can be utilized, the performance of the proposed solution will be further improved. Our future research will consider this aspect.

\section{Competing Interests}

The authors declare that they have no competing interests.

\section{Acknowledgments}

This work was supported by the National Natural Science Foundation of China (Grant no. 61273236), the Jiangsu Provincial Basic Research Program (Natural Science Foundation, Grant no. BK2010239), and the Scientific Research Foundation of Graduate School of Southeast University (no. YBJJ1637).

\section{References}

[1] A. Vu, A. Ramanandan, A. Chen, J. A. Farrell, and M. Barth, "Real-time computer vision/DGPS-aided inertial navigation system for lane-level vehicle navigation," IEEE Transactions on Intelligent Transportation Systems, vol. 13, no. 2, pp. 899-913, 2012.

[2] K. Jo, K. Chu, and M. Sunwoo, "Interacting multiple model filter-based sensor fusion of GPS with in-vehicle sensors for real-time vehicle positioning," IEEE Transactions on Intelligent Transportation Systems, vol. 13, no. 1, pp. 329-343, 2012.

[3] I. Skog and P. Handel, "In-car positioning and navigation technologies-a survey," IEEE Transactions on Intelligent Transportation Systems, vol. 10, no. 1, pp. 4-21, 2009.

[4] Y. Gu, L.-T. Hsu, and S. Kamijo, "GNSS/onboard inertial sensor integration with the aid of 3-D building map for lane-level vehicle self-localization in urban canyon," IEEE Transactions on Vehicular Technology, vol. 65, no. 6, pp. 4274-4287, 2016.

[5] F. Peyret, D. Bétaille, P. Carolina, R. Toledo-Moreo, A. F. GomezSkarmeta, and M. Ortiz, "GNSS autonomous localization: NLOS satellite detection based on 3-D maps," IEEE Robotics and Automation Magazine, vol. 21, no. 1, pp. 57-63, 2014.

[6] Z. Wu, M. Yao, H. Ma, and W. Jia, "Improving accuracy of the vehicle attitude estimation for low-cost INS/GPS integration aided by the GPS-measured course angle," IEEE Transactions on Intelligent Transportation Systems, vol. 14, no. 2, pp. 553-564, 2013.

[7] X. Li, Q. Xu, C. Chan, B. Li, W. Chen, and X. Song, "A hybrid intelligent multisensor positioning methodology for reliable vehicle navigation," Mathematical Problems in Engineering, vol. 2015, Article ID 176947, 13 pages, 2015.

[8] S. Godha and M. E. Cannon, "GPS/MEMS INS integrated system for navigation in urban areas," GPS Solutions, vol. 11, no. 3, pp. 193-203, 2007.

[9] A. Angrisano, S. Gaglione, and C. Gioia, "Performance assessment of aided global navigation satellite system for land navigation," IET Radar, Sonar \& Navigation, vol. 7, no. 6, pp. 671-680, 2013.

[10] Z. Ren and H. Wang, "Fusion estimation algorithm with uncertain noises and its application in navigation system," 
Mathematical Problems in Engineering, vol. 2015, Article ID 218561, 9 pages, 2015.

[11] S.-B. Kim, J.-C. Bazin, H.-K. Lee, K.-H. Choi, and S.-Y. Park, "Ground vehicle navigation in harsh urban conditions by integrating inertial navigation system, global positioning system, odometer and vision data," IET Radar, Sonar and Navigation, vol. 5, no. 8, pp. 814-823, 2011.

[12] R. Toledo-Moreo, M. A. Zamora-Izquierdo, B. Úbeda-Miñarro, and A. F. Gómez-Skarmeta, "High-integrity IMM-EKF-based road vehicle navigation with low-cost GPS/SBAS/INS," IEEE Transactions on Intelligent Transportation Systems, vol. 8, no. 3, pp. 491-511, 2007.

[13] J. Georgy, A. Noureldin, M. J. Korenberg, and M. M. Bayoumi, "Modeling the stochastic drift of a MEMS-based gyroscope in gyro/odometer/GPS integrated navigation," IEEE Transactions on Intelligent Transportation Systems, vol. 11, no. 4, pp. 856-872, 2010.

[14] X. Li, M. Ge, X. Dai et al., "Accuracy and reliability of multiGNSS real-time precise positioning: GPS, GLONASS, BeiDou, and Galileo," Journal of Geodesy, vol. 89, no. 6, pp. 607-635, 2015.

[15] P. D. Groves, "Shadow matching: a new GNSS positioning technique for urban canyons," Journal of Navigation, vol. 64, no. 3, pp. 417-430, 2011.

[16] F. Meng, B. Zhu, and S. Wang, "A new fast satellite selection algorithm for BDS-GPS receivers," in Proceedings of the IEEE Workshop on Signal Processing Systems (SiPS '13), pp. 371-376, Taipei, Taiwan, October 2013.

[17] G. A. McGraw, R. S. Y. Young, K. Reichenauer, J. Stevens, and F. Ventrone, "GPS multipath mitigation assessment of digital beam forming antenna technology in a JPALS dual frequency smoothing architecture," in Proceedings of the Institute of Navigation, National Meeting (NTM '04), pp. 561-572, January 2004.

[18] N. Kubo, T. Suzuki, A. Yasuda, and R. Shibazaki, "An effective method for multipath mitigation under severe multipath environments," in Proceedings of the 18th International Technical Meeting of the Satellite Division of The Institute of Navigation (ION GNSS '05), pp. 2187-2194, Long Beach, Calif, USA, September 2005.

[19] P. Xie and M. G. Petovello, "Measuring GNSS multipath distributions in urban canyon environments," IEEE Transactions on Instrumentation and Measurement, vol. 64, no. 2, pp. 366-377, 2015.

[20] L.-T. Hsu, "Integration of vector tracking loop and multipath mitigation technique and its assessment," in Proceedings of the 26th International Technical Meeting of the Satellite Division of the Institute of Navigation (ION GNSS '13), pp. 3263-3278, Nashville, Tenn, USA, September 2013.

[21] L. Wang, P. D. Groves, and M. K. Ziebart, "Multi-constellation GNSS performance evaluation for urban canyons using large virtual reality city models," Journal of Navigation, vol. 65, no. 3, pp. 459-476, 2012.

[22] L.-T. Hsu, Y. Gu, and S. Kamijo, "NLOS correction/exclusion for GNSS measurement using RAIM and city building models," Sensors, vol. 15, no. 7, pp. 17329-17349, 2015.

[23] D. Betaille, F. Peyret, M. Ortiz, S. Miquel, and L. Fontenay, "A new modeling based on urban trenches to improve GNSS positioning quality of service in cities," IEEE Intelligent Transportation Systems Magazine, vol. 5, no. 3, pp. 59-70, 2013.

[24] M. Obst, S. Bauer, P. Reisdorf, and G. Wanielik, "Multipath detection with 3D digital maps for robust multi-constellation GNSS/INS vehicle localization in urban areas," in Proceedings of the IEEE Intelligent Vehicles Symposium (IV '12), pp. 184-190, Madrid, Spain, June 2012.

[25] A. Noureldin, T. B. Karamat, and J. Georgy, Fundamentals of Inertial Navigation, Satellite-Based Positioning and Their Integration, Springer, 2012.

[26] J. Kong, X. Mao, and S. Li, "BDS/GPS dual systems positioning based on the modified SR-UKF algorithm," Sensors, vol. 16, no. 5, article no. 635, 2016.

[27] K. Jung, J. Kim, J. Kim, E. Jung, and S. Kim, "Positioning accuracy improvement of laser navigation using UKF and FIS," Robotics and Autonomous Systems, vol. 62, no. 9, pp. 1241-1247, 2014.

[28] M. St-Pierre and D. Gingras, "Comparison between the unscented Kalman filter and the extended Kalman filter for the position estimation module of an integrated navigation information system," in Proceedings of the IEEE Intelligent Vehicles Symposium, pp. 831-835, Parma, Italy, June 2004.

[29] P. D. Groves and Z. Jiang, "Height aiding, C/N0 weighting and consistency checking for gnss nlos and multipath mitigation in urban areas," Journal of Navigation, vol. 66, no. 5, pp. 653-669, 2013.

[30] http://www.novatel.com/support/info/documents/564. 


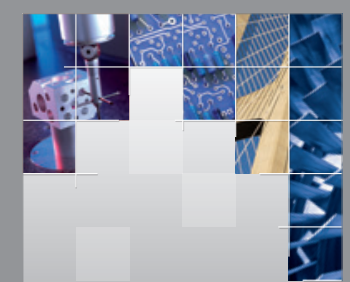

\section{Enfincering}
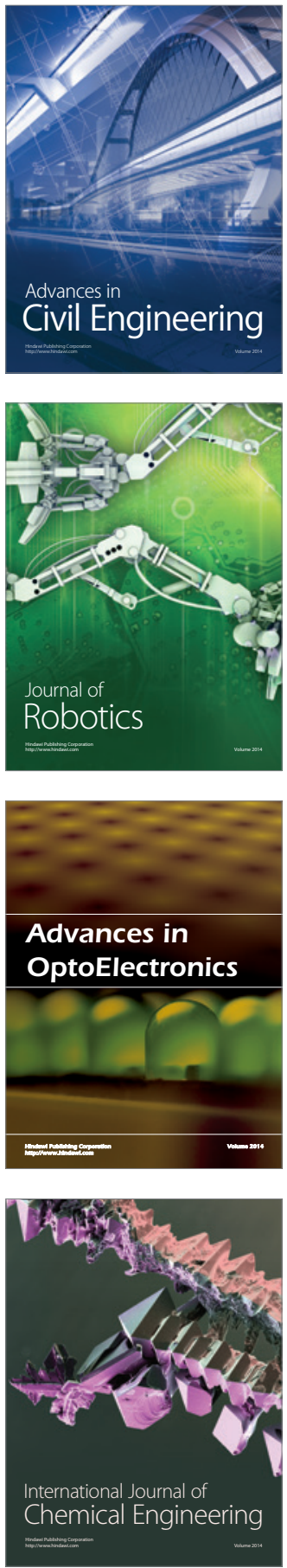

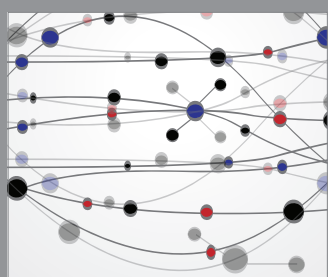

The Scientific World Journal

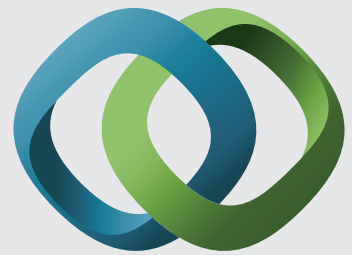

\section{Hindawi}

Submit your manuscripts at

https://www.hindawi.com
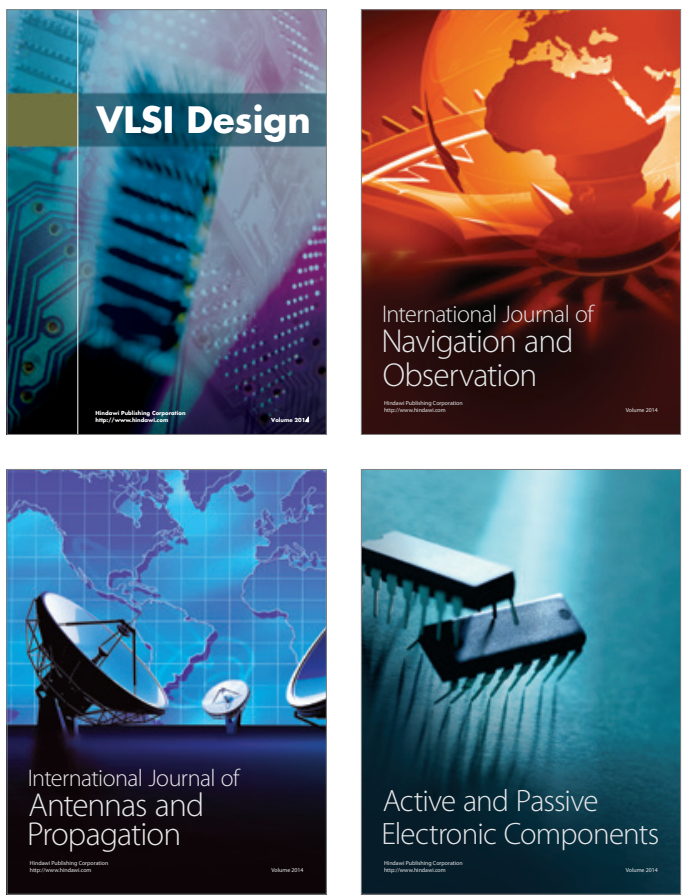
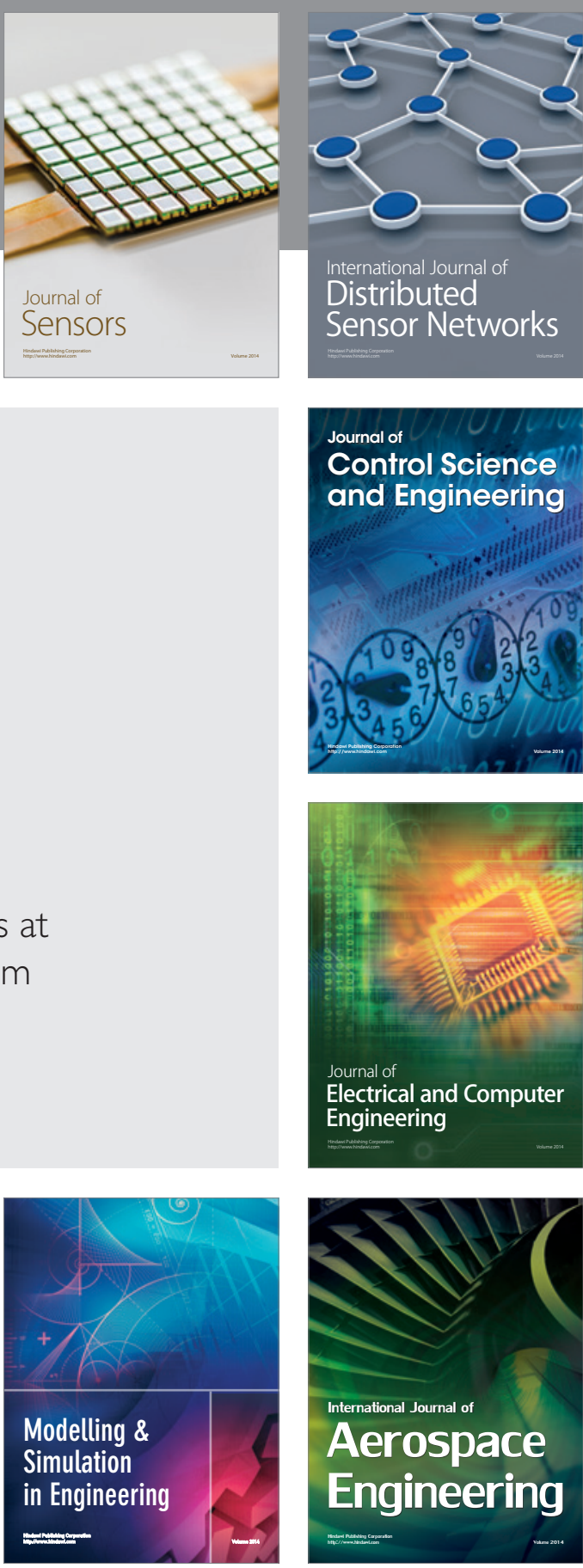

International Journal of

Distributed

Sensor Networks

$-$

Joumal of

Control Science

and Engineering
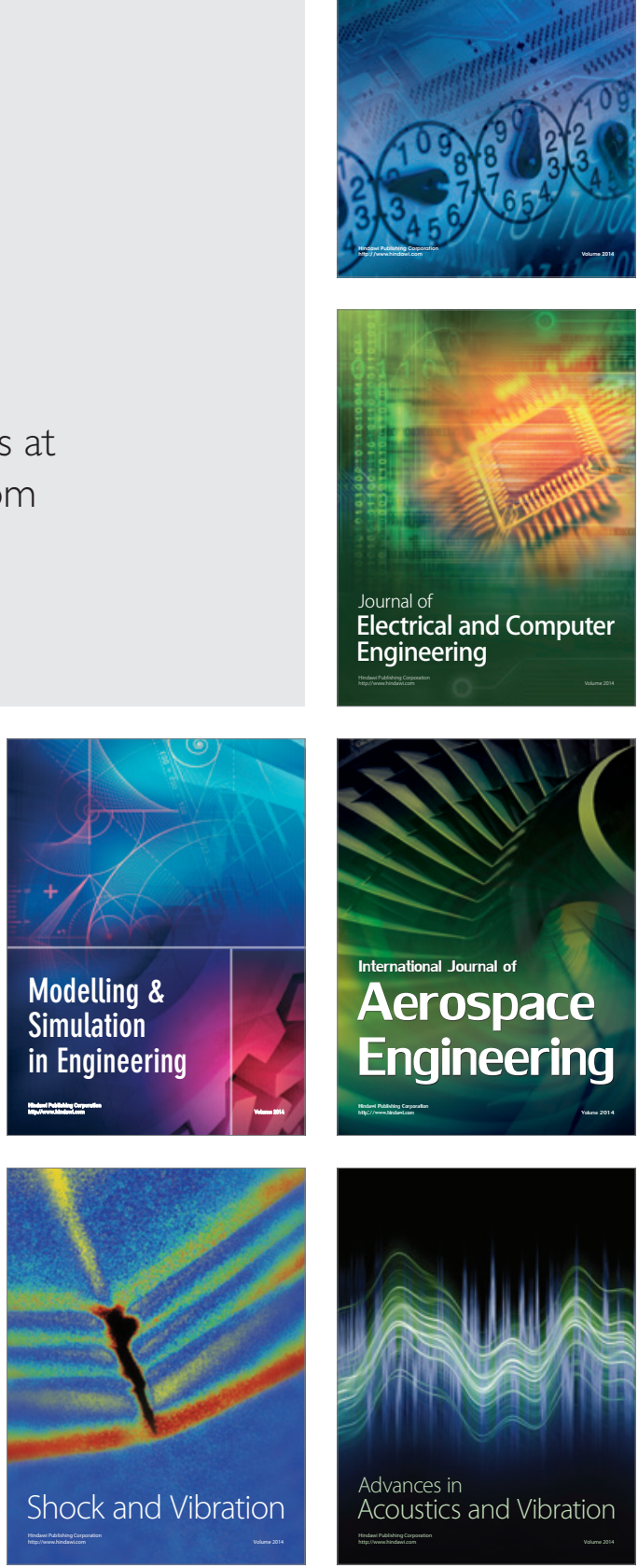